4 5 6 8 9 10 11 12 13 14 15 16 17 18 19 20 21 22 23 24 25 26 27 28 29 30 31 32 8 ( 0 1 2 3 4 15 8

8

\title{
Metabolic reprogramming: an innate cellular defense mechanism against intracellular bacteria?
}

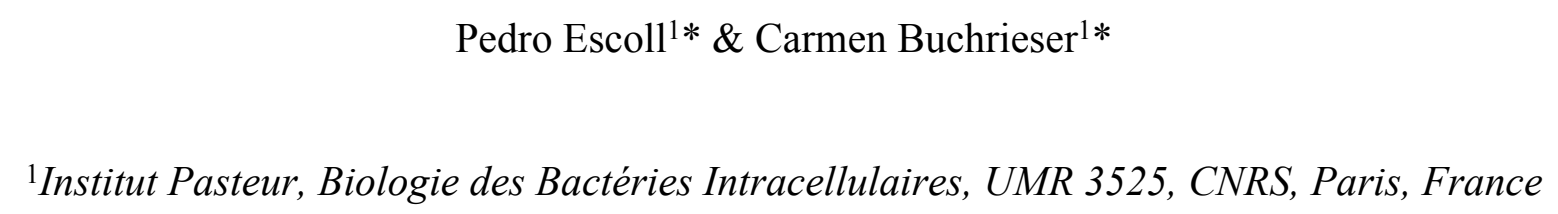
6

7 Short title: Immunometabolism and intracellular bacteria

9 Key words: Intracellular bacteria, metabolism, innate immune cells, host-pathogen 0 inetractions

*For correspondence:

Pedro Escoll and Carmen Buchrieser Institut Pasteur, Biologie des Bactéries Intracellulaires 28, rue du Dr. Roux, 75724 Paris Cedex 15, France Tel: (33-1)-45-68-83-72 Fax: (33-1)-45-68-87-86 E-mail: pescoll@pasteur.fr and cbuch@pasteur.fr 


\section{Abstract}

35 The limited metabolic resources of a cell represent an intriguing "conflict of interest" during 36 host-pathogen interactions, as the battle for nutrients might determine the outcome of an 37 infection. To adapt their metabolic needs, innate immune cells such as monocytes, 38 macrophages or dendritic cells reprogram their metabolism upon activation by microbial 39 compounds. In turn, infection by intracellular bacteria provokes metabolic alterations of the 40 host cell that benefit the pathogen. Here we discuss the state-of-the-art knowledge on 41 metabolic reprogramming of host cells upon activation or infection with intracellular bacteria. 42 The study of the host- and pathogen-driven metabolic alterations that seem to co-exist during 43 infection is an emerging field that will define the metabolic pathways that might be targeted 44 to combat infection. 
47 Cellular metabolism comprises the controlled biochemical processes that occur within a cell to maintain life. It is generally divided in, anabolic processes (the build-up of macromolecules) and catabolic processes (the breakdown of macromolecules). To maintain constant cellular conditions in response to changes in the environment (homeostasis) a cell regulates its specific needs by the fine-tuning of these metabolic pathways.

During host-pathogen interactions, host and pathogen fight for the nutrients needed for cellular catabolism, as resources are generally limited [1,2]. Obtaining energy and ready-touse basic molecules is key to build up new cellular components, to grow and reproduce, or to respond to environmental changes. Thus the result of this metabolic clash is key for the outcome of host-pathogen interactions and the infection process.

Cellular metabolism is one of the most complex and intricate cellular systems. To simplify, two main catabolic programs work to obtain energy, "glycolysis" and "oxidative phosphorylation" (OXPHOS) (Figure 1A). During glycolysis that is performed in the cytosol, cells convert glucose into pyruvate by anaerobic glycolysis and then pyruvate is fermented to lactate. Thereby 2 ATP molecules are generated per molecule of glucose. During OXPHOS the pyruvate generated by anaerobic glycolysis is routed to mitochondria where it is oxidized to $\mathrm{CO}_{2}$ via the tricarboxylic acid (TCA) cycle. The released energy (stored as NADH) is used by the electron transport chain (ETC) to pump protons across the membrane. This generates an electrochemical gradient used to synthesize ATP by the mitochondrial $F_{1}-F_{O}-A T P a s e$. Under the OXPHOS program, the cell produces 36 ATPs per molecule of glucose [3]. Interestingly, many proliferating cells (such as embryonic stem cells, cancer cells or activated T-cells) prefer the glycolytic program although this metabolic pathway is less efficient for ATP production as compared to OXPHOS. In contrast, tumour cells convert most of the absorbed glucose to lactate even under oxygen-rich conditions, a program that was termed "aerobic glycolysis" or the "Warburg effect" (Figure 1B) [4]. Thus elevated glucose uptake and lactate secretion are metabolic hallmarks of solid tumours [5,6]. Although the question why cancer cells prefer a "less efficient" metabolic pathway to grow is still a matter of debate, it seems that aerobic glycolysis coupled to fermentation is a fast process, easy to regulate, that fulfils all metabolic requirements of a proliferating cell. The Warburg program maximizes biosynthetic pathways by redirecting cytosolic glycolysis and the mitochondrial TCA cycle to the biosynthesis of nucleotides, amino acids and lipids (Figure 1B). The fast withdrawal of pyruvate and TCA intermediates in the mitochondria, which are normally 
dedicated to the OXPHOS program and their redirection to biosynthetic pathways, explains the reduction of oxygen consumption after the switch to the Warburg metabolism. Thus Warburg metabolism seems to be induced to reprogram cellular metabolism towards macromolecular synthesis.

Reprogramming to Warburg-like metabolism has been observed during activation of diverse immune cells and, interestingly, also during infection of human cells by intracellular bacterial pathogens such as Mycobacterium tuberculosis [7-11], Legionella pneumophila [12], Brucella abortus [13], Chlamydia trachomatis [14,15] or C. pneumoniae [16]. Although the Warburg program in proliferating T-cells and cancer cells can be seen as program necessary for proliferation, the induction of the Warburg program in infected cells raises intriguing questions. Why non-proliferating immune cells, such as monocytes, dendritic cells (DCs) or macrophages, switch to a Warburg-like metabolism during infection? What is the impact of such metabolic changes on the pathogen that obtains its nutrients only from the infected host cell? Is metabolic reprogramming beneficial or detrimental for intracellular bacteria? Are these metabolic alterations driven by the host cell itself in response to bacteria, as a defence mechanism, or are they induced by the pathogen for its own benefit?

\section{Non-proliferating immune cells reprogram metabolic pathways upon stimulation with microbial compounds}

Monocytes, DCs and macrophages are differentiated, non-proliferative, innate immune cells that, once activated by microbial stimuli, orchestrate the activation of other immune cells through the secretion of immuno-regulatory cytokines, but they also increase glycolytic fluxes and modulate their metabolism.

In DCs, glucose uptake is increased and accompanied by lactate production shortly after their activation by microbial compounds that engage Toll-like receptors (TLRs) such as bacterial lipoplysacharide (LPS). After TLR stimulation, a signalling cascade involving TBK1, IKK $\square$ and Akt promotes the association of the glycolytic enzyme hexokinase-2 (HK2) with mitochondria increasing its enzymatic activity and therefore the glycolytic flux, however, cellular ATP is still provided by OXPHOS [17]. At these early time points, glycolysis supplies DCs with citrate, which fuels the synthesis of fatty acids that are required by activated DCs to increase the size of the endoplasmic reticulum (ER) and the Golgi apparatus [17]. Thus, the fast and early metabolic switch of DCs to a glycolytic program due to the stimulation with microbial compounds is linked to fatty acid synthesis and the adoption of the 
112 secretory state. At $24 \mathrm{~h}$ of DC activation by LPS, iNOS-derived NO inhibits cellular

113 OXPHOS, which almost disappears and DCs rely exclusively on glycolysis, a Warburg-like 114 metabolic program is installed [18].

115 In murine bone-marrow-derived macrophages (BMDMs), metabolic reprogramming to a 116 glycolytic Warburg-like program is necessary for transcription of the pro-inflammatory 117 cytokine IL1 $\beta$. Upon LPS stimulation, BMDMs show a broken TCA cycle and intermediates, 118 such as succinate or citrate, accumulate [19]. Furthermore, LPS induces the up-regulation of 119 pyruvate kinase M2 (PKM2) dimer and tetramer levels. In its enzymatically inactive form 120 (dimers), increased PKM2 induces succinate accumulation [20]. PKM2 dimers also migrate to 121 the nucleus where they induce PKM2-mediated transcription of IL1 $\beta$. In its enzymatically 122 active form (tetramers), LPS-induction of PKM2 also increases the rate of glycolytic fluxes, 123 as the reaction catalysed by PKM2 is the rate-limiting step of glycolysis. Accumulated 124 succinate stabilizes HIF1 $\alpha$, which can now translocate to the nucleus and, together with 125 PKM2 dimers, drive IL1 $\beta$ gene expression. Succinate in mitochondria is oxidized by succinate dehydrogenase and leads to the reverse flow of the ETC, which produces mitochondrial reactive oxygen species (mROS) that also induce HIF $1 \alpha$-mediated IL1 $\beta$ mRNA expression [21]. Accumulation of citrate is due to the LPS-induced expression of the mitochondrial citrate carrier, which diverts citrate from the mitochondrial TCA cycle to the cytoplasm. Cytoplasmic citrate is then used to generate NO, ROS and prostaglandins by BMDMs, as well as to produce the antimicrobial metabolite itaconate [22-24].

132 Taken together, exposure of murine macrophages to bacterial LPS reprograms their 133 metabolism from OXPHOS to glycolysis, rewiring TCA intermediates such as citrate and 134 succinate to biosynthetic pathways. As itaconate has direct antimicrobial proprieties against a 135 wide range of intracellular bacteria, such as M.tuberculosis, Salmonella enterica or 136 L. pneumophila [25], the redirection of citrate for the synthesis of itaconate represents a 137 prominent example of how metabolic reprogramming of activated macrophages becomes a 138 defence mechanism against infection by intracellular bacteria. In addition to its antibacterial 139 proprieties, itaconate has also immunoregulatory functions during infection due to its 140 inhibitory actions on the production of mROS and certain cytokines and chemokines as 141 shown for BMDMs infected with $S$. enterica serovar Typhimurium [26], and for mice 142 infected with M. tuberculosis [27].

143 Recently it was shown that human monocytes isolated from blood also shift to a Warburg144 like metabolism when stimulated with LPS, but not upon stimulation with the TLR2 ligand 
145 Pam3Cys (P3C) or whole-pathogen lysates. Indeed, in P3C stimulated monocytes, increased

146 glycolysis was accompanied by increased OXPHOS, which was required for retention of their 147 phagocytic capacity and cytokine production [28]. Elevated succinate, itaconate and citrate 148 levels were only observed in LPS-stimulated monocytes, but not in P3C-stimulated 149 monocytes. Interestingly, exposure of human monocytes to M. tuberculosis whole cell lysates 150 did not affect OXPHOS, in contrast to infection of monocytes with living M. tuberculosis or 151 after exposure to LPS as OXPHOS reduction was observed [9,28]. Moreover, exposure of 152 monocytes to Escherichia coli or Staphylococcus aureus whole-cell lysates leads to increased 153 OXPHOS [28], suggesting that heterogeneous metabolic responses may be elicited depending 154 on the specific pathogen and TLR activation pattern involved.

155 These results indicate that metabolic reprogramming of innate immune cells upon stimulation with microbial components rewires cellular metabolism to biosynthetic pathways that drive growth of cell size and production and secretion of cytokines and antimicrobial compounds. It is critical to clarify whether these metabolic alterations are stimuli-specific (LPS vs. P3C), cell-type specific (macrophages vs. monocytes vs. DCs), host-species specific (mouse vs. human) and/or bacterial-species specific (S. aureus vs. M. tuberculosis).

\section{Intracellular bacteria reprogram the host metabolism to a Warburg-like metabolism}

M. tuberculosis [7-11], L. pneumophila [12], B. abortus [13], C. trachomatis [14,15], C. pneumoniae [16] or Listeria monocytogenes [29,30], were reported to modulate the metabolism of their host cells to a Warburg-like metabolism [31]. Yet only few studies addressed the metabolism of primary cells during infection thus one must be careful with the interpretation of metabolic changes as most cancer cell lines have an enhanced Warburg metabolism due to their tumour origin [31]. This has been demonstrated for L. monocytogenes, as high induction of glucose uptake and glycolysis was only observed in primary BMDMs, and not in the murine macrophage-like cells line J774A.1. However, intracellular bacteria

171 replicated to five fold higher numbers within the cancer cell line than in primary cells, 172 suggesting that the Warburg metabolism exhibited by cell lines might promote bacterial 173 replication [29].

174 The changes to a Warburg-like metabolism induced by many intracellular bacteria come in 175 different styles (Figure 3). Chlamydia trachomatis infection of primary human umbilical vein 176 endothelial cells leads to a specific Warburg-like program. Cellular glucose uptake and the 177 phosphate pentose pathway (PPP), a nucleotide biosynthetic pathway derived from the 
glycolytic flux are increased [15] (Figure 3A,Chl). The infection-dependent upregulation of the PPP supplies the pathogen with nucleotides for intracellular replication, which serve as nutrients together with amino acids, and lipids that $C$. trachomatis needs to sequester from the host cell [32]. Thus, the induction of this Warburg-like program during infection seems to benefit the pathogen.

L. pneumophila induces a Warburg-like metabolism characterized by the upregulation of glycolysis and reduction of OXPHOS dependent on the injection of the bacterial effector MitF in the host cell that induces reduction of OXPHOS through fragmentation of mitochondrial networks [12]. This metabolic reprogramming of infected cells to a Warburglike program appears essential for bacterial growth and might be related to the need of amino acids, the main source of energy for growing L. pneumophila [3,33], which can be synthesized from redirected glycolytic and/or TCA intermediates (Figure 3B, $L p$ ).

M. tuberculosis infection induces a Warburg-like program when infecting human primary macrophages, that is characterized by decreased OXPHOS and upregulation of glucose uptake and glycolysis coupled with the redirection of glycolytic intermediates to the synthesis of large lipid bodies that accumulate in the macrophage (Figure $3 \mathrm{C}, M t b$ ). These lipid bodies, together with the lactate produced by glycolysis, serve as nutrients for intracellular growth of the pathogen [34-36], suggesting that the biosynthetic role of the Warburg-like program benefits the intracellular replication of M. tuberculosis.

Although it is not clear yet whether Salmonella enterica, induces a Warburg-like metabolism in the host cells or not, it has been reported that $S$. enterica modulates the metabolism of the cell it infects. During in vivo infection of mice with $S$. enterica serovar typhimuriumOXPHOS is completely shut-off [37], while glycolysis might be induced [38]. However, the most prominent metabolic alteration induced by S.typhimurium seems the piracy of glucose by the pathogen. In mouse BMDMs Salmonella disrupts glycolytic fluxes to reroute glucose from host macrophages to its vacuole (Figure 3A, St) and thereby this pathogen reduces host glucose availability [39,40]. Interestingly, inhibition of glycolytic fluxes creates a unique metabolic defect that activates the NLRP3 inflammasome [40].

Intracellular bacteria modulate autophagy, a catabolic program and a cellular defense system

Another important metabolic response to infection is the cellular process known as autophagy, a self-degradative process that is key for balancing sources of energy in response 
211 to nutrient stress and to remove intracellular pathogens in response to infection [41-43].

212 Interestingly, autophagy also regulates cytokine production and secretion in immune cells 213 upon microbial stimulation $[44,45]$.

214 Host-driven and pathogen-driven alterations of autophagy occur during infection with 215 intracellular bacteria. As host-induced autophagy degrades invading pathogens, evasion of 216 autophagy is key for pathogens such as M. tuberculosis, S. typhimurium or L. pneumophila 217 that inhibit autophagy initiation upstream of autophagosome formation, or Shigella flexneri 218 that evades autophagy recognition by masking the bacterial surface [46-49]. Interestingly,

219 L. pneumophila encodes two secreted effectors, RavZ and LpSPL that inhibit autophagosome 220 formation at two different stages [47,50]. Other intracellular bacteria such as Anaplasma 221 phagocytophilum, Yersinia pseudotuberculosis, Coxiella burnetii and Francisella tularensis 222 hijack autophagosomes during infection to redirect the by-products of the autophagic 223 degradation of cellular components to their vacuoles for their own nutritional use, which 224 finally promotes bacterial replication [51]. For instance, A. phagocytophilum secrets the 225 effector Ats-1 to induce autophagosome formation to obtain nutrients contained in them [52], 226 showing that pathogens may activate autophagy also for their benefit.

227 Taken together, these results suggest that modulation of autophagy during bacterial 228 infection is host- as well as pathogen-driven and leads to alterations of the metabolism and the 229 immune defences of the infected cell.

\section{Concluding remarks}

232 Some metabolic host responses, such as activation of the HIF1 $\alpha$ axis, commonly occur in 233 macrophages and other cell types upon bacterial infection [53]. However, pathogen specific 234 reprogramming of the cellular metabolism [28] and metabolic alterations triggered by 235 bacterial effectors [12], which seem beneficial for the pathogen, also exist. A recurrent debate 236 is whether the observed metabolic changes in infected cells are host-driven in response to 237 bacterial invasion, or driven by the pathogen to benefit its intracellular survival and 238 replication. As some metabolic alterations such as autophagy modulation or induction of 239 Warburg-like programs, seem dependent on bacterial effectors and beneficial for the pathogen, 240 both processes seem to happen in parallel and thus the observed metabolic phenotypes during 241 infection are a mixture of host-driven and pathogen-driven metabolic reprogramming, reflecting the battle for resources in an infected cell. 
243 Furthermore, some pathogen-mediated metabolic alterations during infection seem to trigger

244 host immune responses. An example is the NLRP3 inflammasome activation following the 245 inhibition of glycolytic fluxes at the level of GAPDH by S. enterica [40], or the binding of

246 bacterial sugars to the glycolytic enzyme HK2, a member of the sugar kinase family that

247 binds glucose and surprisingly triggers NLRP3 inflammasome activation when binding

248 bacterial peptidoglycan-derived N-acetylglucosamine [54].

249 Although many puzzling enigmas remain to be clarified, a novel aspect may emerge from 250 the "immune surveillance hypothesis" [55]. While this hypothesis defends that pathogen251 mediated disruption of host physiology leads to immune activation, we think that an 252 additional novel aspect should be added: specific checkpoints might exist along cellular 253 metabolic pathways that, when altered, trigger immune responses such as inflammasome 254 activation. We thus suggest the term "Danger-Associated Metabolic Modifications" 255 (DAMMs) for alterations in the metabolism of host cells that trigger defence responses. 256 OXPHOS dysfunction, altered glycolytic fluxes or piracy of metabolic intermediates induced 257 by intracellular bacteria might be DAMMs linking pathogen-driven and host-driven metabolic 258 reprogramming. Future work should shed light on these exciting and complex host-pathogen 259 interactions.

261 Declarations of interest: none.

263 Acknowledgments

264 This work was supported by the Institut Pasteur and the Agence National de Recherche [grant 265 number ANR-10-LABX-62-IBEID]. 
1. Eisenreich W, Rudel T, Heesemann J, Goebel W: To Eat and to Be Eaten: Mutual Metabolic Adaptations of Immune Cells and Intracellular Bacterial Pathogens upon Infection. Front Cell Infect Microbiol 2017, 7:316.

** Comprehnsive and excellent review describing how pathogenic, intracellular bacteria and immune cells mutually modulate the metabolism. Together with reference 27 , a complete compendium of the state-of-the-art of the field.

2. Olive AJ, Sassetti CM: Metabolic crosstalk between host and pathogen: sensing, adapting and competing. Nat Rev Microbiol 2016, 14:221-234.

3. Escoll P, Buchrieser $\mathrm{C}$ : Metabolic reprogramming of host cells upon bacterial infection: Why shift to a Warburg-like metabolism? FEBS J 2018.

4. Warburg O: The metabolism of carcinoma cells. The Journal of Cancer Research 1925, 9:148-163.

5. Koppenol WH, Bounds PL, Dang CV: Otto Warburg's contributions to current concepts of cancer metabolism. Nat Rev Cancer 2011, 11:325-337.

6. Ward PS, Thompson CB: Metabolic reprogramming: a cancer hallmark even warburg did not anticipate. Cancer Cell 2012, 21:297-308.

7. Appelberg R, Moreira D, Barreira-Silva P, Borges M, Silva L, Dinis-Oliveira RJ, Resende M, Correia-Neves M, Jordan MB, Ferreira NC, et al.: The Warburg effect in mycobacterial granulomas is dependent on the recruitment and activation of macrophages by interferon-gamma. Immunology 2015, 145:498-507.

8. Gleeson LE, Sheedy FJ, Palsson-McDermott EM, Triglia D, O'Leary SM, O'Sullivan MP, O'Neill LA, Keane J: Cutting Edge: Mycobacterium tuberculosis Induces Aerobic Glycolysis in Human Alveolar Macrophages That Is Required for Control of Intracellular Bacillary Replication. J Immunol 2016, 196:2444-2449.

9. Lachmandas E, Beigier-Bompadre M, Cheng SC, Kumar V, van Laarhoven A, Wang X, Ammerdorffer A, Boutens L, de Jong D, Kanneganti TD, et al.: Rewiring cellular metabolism via the AKT/mTOR pathway contributes to host defence against Mycobacterium tuberculosis in human and murine cells. Eur J Immunol 2016, 46:2574-2586.

10. Shi L, Salamon H, Eugenin EA, Pine R, Cooper A, Gennaro ML: Infection with Mycobacterium tuberculosis induces the Warburg effect in mouse lungs. Sci Rep 2015, 5:18176.

11. Somashekar BS, Amin AG, Rithner CD, Troudt J, Basaraba R, Izzo A, Crick DC, Chatterjee D: Metabolic profiling of lung granuloma in Mycobacterium tuberculosis infected guinea pigs: ex vivo $1 \mathrm{H}$ magic angle spinning NMR studies. $J$ Proteome Res 2011, 10:4186-4195.

12. Escoll P, Song OR, Viana F, Steiner B, Lagache T, Olivo-Marin JC, Impens F, Brodin P, Hilbi H, Buchrieser $\mathrm{C}$ : Legionella pneumophila Modulates Mitochondrial Dynamics to Trigger Metabolic Repurposing of Infected Macrophages. Cell Host Microbe 2017, 22.

13. Czyz DM, Willett JW, Crosson S: Brucella abortus Induces a Warburg Shift in Host Metabolism That Is Linked to Enhanced Intracellular Survival of the Pathogen. $J$ Bacteriol 2017, 199.

14. Ojcius DM, Degani H, Mispelter J, Dautry-Varsat A: Enhancement of ATP levels and glucose metabolism during an infection by Chlamydia. NMR studies of living cells. J Biol Chem 1998, 273:7052-7058.

15. Siegl C, Prusty BK, Karunakaran K, Wischhusen J, Rudel T: Tumor suppressor p53 alters host cell metabolism to limit Chlamydia trachomatis infection. Cell Rep 2014, 9:918-929. 
16. Rupp J, Gieffers J, Klinger M, van Zandbergen G, Wrase R, Maass M, Solbach W, Deiwick J, Hellwig-Burgel T: Chlamydia pneumoniae directly interferes with HIF1alpha stabilization in human host cells. Cell Microbiol 2007, 9:2181-2191.

17. Everts B, Amiel E, Huang SC, Smith AM, Chang CH, Lam WY, Redmann V, Freitas TC, Blagih J, van der Windt GJ, et al.: TLR-driven early glycolytic reprogramming via the kinases TBK1-IKKvarepsilon supports the anabolic demands of dendritic cell activation. Nat Immunol 2014, 15:323-332.

18. Everts B, Amiel E, van der Windt GJ, Freitas TC, Chott R, Yarasheski KE, Pearce EL, Pearce EJ: Commitment to glycolysis sustains survival of NO-producing inflammatory dendritic cells. Blood 2012, 120:1422-1431.

19. Tannahill GM, Curtis AM, Adamik J, Palsson-McDermott EM, McGettrick AF, Goel G, Frezza C, Bernard NJ, Kelly B, Foley NH, et al.: Succinate is an inflammatory signal that induces IL-1beta through HIF-1alpha. Nature 2013, 496:238-242.

20. Palsson-McDermott EM, Curtis AM, Goel G, Lauterbach MA, Sheedy FJ, Gleeson LE, van den Bosch MW, Quinn SR, Domingo-Fernandez R, Johnston DG, et al.: Pyruvate kinase $\mathrm{M} 2$ regulates Hif-1alpha activity and $\mathrm{IL}-1$ beta induction and is a critical determinant of the warburg effect in LPS-activated macrophages. Cell Metab 2015, 21:65-80.

**Outstanding article that elegantly deciphers the role of PKM2 dimers and tetramers in the regulation of the Warburg-like metabolism induced by LPS in macrophages, and its consequence on infection with Mycobacterium tuberculosis or Salmonella typhimurium

21. Mills EL, Kelly B, Logan A, Costa ASH, Varma M, Bryant CE, Tourlomousis P, Dabritz JHM, Gottlieb E, Latorre I, et al.: Succinate Dehydrogenase Supports Metabolic Repurposing of Mitochondria to Drive Inflammatory Macrophages. Cell 2016, 167:457-470 e413.

22. Infantino V, Convertini P, Cucci L, Panaro MA, Di Noia MA, Calvello R, Palmieri F, Iacobazzi $\mathrm{V}$ : The mitochondrial citrate carrier: a new player in inflammation. Biochem J 2011, 438:433-436.

23. Jha AK, Huang SC, Sergushichev A, Lampropoulou V, Ivanova Y, Loginicheva E, Chmielewski K, Stewart KM, Ashall J, Everts B, et al.: Network integration of parallel metabolic and transcriptional data reveals metabolic modules that regulate macrophage polarization. Immunity 2015, 42:419-430.

24. Michelucci A, Cordes T, Ghelfi J, Pailot A, Reiling N, Goldmann O, Binz T, Wegner A, Tallam A, Rausell A, et al.: Immune-responsive gene 1 protein links metabolism to immunity by catalyzing itaconic acid production. Proc Natl Acad Sci U S A 2013, 110:7820-7825.

25. Luan HH, Medzhitov R: Food Fight: Role of Itaconate and Other Metabolites in Antimicrobial Defense. Cell Metab 2016, 24:379-387.

26. Lampropoulou V, Sergushichev A, Bambouskova M, Nair S, Vincent EE, Loginicheva E, Cervantes-Barragan L, Ma X, Huang SC, Griss T, et al.: Itaconate Links Inhibition of Succinate Dehydrogenase with Macrophage Metabolic Remodeling and Regulation of Inflammation. Cell Metab 2016, 24:158-166.

27. Nair S, Huynh JP, Lampropoulou V, Loginicheva E, Esaulova E, Gounder AP, Boon ACM, Schwarzkopf EA, Bradstreet TR, Edelson BT, et al.: Irg1 expression in myeloid cells prevents immunopathology during $M$. tuberculosis infection. $J$ Exp Med 2018, 215:1035-1045.

28. Lachmandas E, Boutens L, Ratter JM, Hijmans A, Hooiveld GJ, Joosten LA, Rodenburg RJ, Fransen JA, Houtkooper RH, van Crevel R, et al.: Microbial stimulation of different Toll-like receptor signalling pathways induces diverse metabolic programmes in human monocytes. Nat Microbiol 2016, 2:16246. 
** Important article showing for the first time the heterogenous nature of metabolic responses of human primary monocytes to different TLR stimuli or whole-bacterial cell lysates.

29. Gillmaier N, Gotz A, Schulz A, Eisenreich W, Goebel W: Metabolic responses of primary and transformed cells to intracellular Listeria monocytogenes. PLoS One 2012, 7:e52378.

30. Stavru F, Bouillaud F, Sartori A, Ricquier D, Cossart P: Listeria monocytogenes transiently alters mitochondrial dynamics during infection. Proc Natl Acad Sci U $S$ A 2011, 108:3612-3617.

31. Eisenreich W, Heesemann J, Rudel T, Goebel W: Metabolic host responses to infection by intracellular bacterial pathogens. Front Cell Infect Microbiol 2013, 3:24.

32. Saka HA, Valdivia RH: Acquisition of nutrients by Chlamydiae: unique challenges of living in an intracellular compartment. Curr Opin Microbiol 2010, 13:4-10.

33. Hauslein I, Sahr T, Escoll P, Klausner N, Eisenreich W, Buchrieser C: Legionella pneumophila CsrA regulates a metabolic switch from amino acid to glycerolipid metabolism. Open Biol 2017, 7.

34. Billig S, Schneefeld M, Huber C, Grassl GA, Eisenreich W, Bange FC: Lactate oxidation facilitates growth of Mycobacterium tuberculosis in human macrophages. Sci Rep 2017, 7:6484.

35. Kim MJ, Wainwright HC, Locketz M, Bekker LG, Walther GB, Dittrich C, Visser A, Wang W, Hsu FF, Wiehart U, et al.: Caseation of human tuberculosis granulomas correlates with elevated host lipid metabolism. EMBO Mol Med 2010, 2:258-274.

36. Singh V, Jamwal S, Jain R, Verma P, Gokhale R, Rao KV: Mycobacterium tuberculosisdriven targeted recalibration of macrophage lipid homeostasis promotes the foamy phenotype. Cell Host Microbe 2012, 12:669-681.

37. Liu X, Lu R, Xia Y, Sun J: Global analysis of the eukaryotic pathways and networks regulated by Salmonella typhimurium in mouse intestinal infection in vivo. $B M C$ Genomics 2010, 11:722.

38. Ding K, Zhang C, Li J, Chen S, Liao C, Cheng X, Yu C, Yu Z, Jia Y: cAMP Receptor Protein of Salmonella enterica Serovar Typhimurium Modulate Glycolysis in Macrophages to Induce Cell Apoptosis. Curr Microbiol 2018.

39. Bowden SD, Rowley G, Hinton JC, Thompson A: Glucose and glycolysis are required for the successful infection of macrophages and mice by Salmonella enterica serovar typhimurium. Infect Immun 2009, 77:3117-3126.

40. Sanman LE, Qian Y, Eisele NA, Ng TM, van der Linden WA, Monack DM, Weerapana E, Bogyo $\mathrm{M}$ : Disruption of glycolytic flux is a signal for inflammasome signaling and pyroptotic cell death. Elife 2016, 5:e13663.

*Interesting paper showing how disruption of metabolic pathways by bacterial-induced piracy of nutrients leds to inflammasome activation.

41. Escoll P, Rolando M, Buchrieser C: Modulation of Host Autophagy during Bacterial Infection: Sabotaging Host Munitions for Pathogen Nutrition. Front Immunol 2016, 7:81.

42. Martinez J, Verbist K, Wang R, Green DR: The relationship between metabolism and the autophagy machinery during the innate immune response. Cell Metab 2013, 17:895-900.

43. Rathmell JC: Metabolism and autophagy in the immune system: immunometabolism comes of age. Immunol Rev 2012, 249:5-13.

44. Harris J: Autophagy and IL-1 Family Cytokines. Front Immunol 2013, 4:83.

45. Kunz TC, Viana F, Buchrieser C, Escoll P: Manipulation of Autophagy by Bacterial Pathogens Impacts Host Immunity. Curr Issues Mol Biol 2018, 25:81-98. 
46. Ogawa M, Yoshimori T, Suzuki T, Sagara H, Mizushima N, Sasakawa C: Escape of intracellular Shigella from autophagy. Science 2005, 307:727-731.

47. Rolando M, Escoll P, Nora T, Botti J, Boitez V, Bedia C, Daniels C, Abraham G, Stogios PJ, Skarina T, et al.: Legionella pneumophila S1P-lyase targets host sphingolipid metabolism and restrains autophagy. Proc Natl Acad Sci U S A 2016, 113:19011906.

48. Shin DM, Jeon BY, Lee HM, Jin HS, Yuk JM, Song CH, Lee SH, Lee ZW, Cho SN, Kim $\mathrm{JM}$, et al:: Mycobacterium tuberculosis eis regulates autophagy, inflammation, and cell death through redox-dependent signaling. PLoS Pathog 2010, 6:e1001230.

49. Tattoli I, Sorbara MT, Vuckovic D, Ling A, Soares F, Carneiro LA, Yang C, Emili A, Philpott DJ, Girardin SE: Amino acid starvation induced by invasive bacterial pathogens triggers an innate host defense program. Cell Host Microbe 2012, 11:563-575.

50. Choy A, Dancourt J, Mugo B, O'Connor TJ, Isberg RR, Melia TJ, Roy CR: The Legionella effector RavZ inhibits host autophagy through irreversible Atg8 deconjugation. Science 2012, 338:1072-1076.

51. Steele $\mathrm{S}$, Brunton $\mathrm{J}$, Kawula $\mathrm{T}$ : The role of autophagy in intracellular pathogen nutrient acquisition. Front Cell Infect Microbiol 2015, 5:51.

52. Niu H, Xiong Q, Yamamoto A, Hayashi-Nishino M, Rikihisa Y: Autophagosomes induced by a bacterial Beclin 1 binding protein facilitate obligatory intracellular infection. Proc Natl Acad Sci U S A 2012, 109:20800-20807.

53. Werth N, Beerlage C, Rosenberger C, Yazdi AS, Edelmann M, Amr A, Bernhardt W, von Eiff $\mathrm{C}$, Becker $\mathrm{K}$, Schafer A, et al.: Activation of hypoxia inducible factor 1 is a general phenomenon in infections with human pathogens. PLoS One 2010, 5:e11576.

54. Wolf AJ, Reyes CN, Liang W, Becker C, Shimada K, Wheeler ML, Cho HC, Popescu NI, Coggeshall KM, Arditi M, et al.: Hexokinase Is an Innate Immune Receptor for the Detection of Bacterial Peptidoglycan. Cell 2016, 166:624-636.

**Outstanding paper showing the immune surveillance function of the metabolic enzyme Hexokinase.

55. Pukkila-Worley R: Surveillance Immunity: An Emerging Paradigm of Innate Defense Activation in Caenorhabditis elegans. PLoS Pathog 2016, 12:e1005795. 
Figure 1. General cellular metabolism (A) and the metabolic Warburg program (B). pathways appear in violet. Blue electron transport chain (ETC): active optimal OXPHOS; pink ETC: reduced OXPHOS.

Figure 2. Metabolic reprogramming of innate immune cells upon exposure to TLR4 or TLR2 ligands. (A) In DCs, TLR4 engagement by LPS increases glycolysis through the activation of a TBK1-Akt-HK2 signalling axis. Glycolysis supplies DCs with citrate, which fuels the synthesis of fatty acids that are required by activated DCs to increase their secretory capacities. (B) In BMDM, LPS induces dimer and tetramer levels of PKM2. Increased PKM2 dimers lead to succinate accumulation and dimers also migrate to the nucleus, where they induce IL1 $\beta$ transcription. LPS-induction of PKM2 tetramers increases the rate of glycolysis.

464 A broken TCA cycle leads to OXPHOS reduction and succinate and citrate accumulation. Accumulated succinate stabilizes HIF1 $\alpha$ that can be translocated to the nucleus and drive IL1 $\beta$ gene expression. In mitochondria, succinate oxidation leads to mROS production and subsequent HIF1 $\alpha$-mediated IL1 $\beta$ mRNA expression. Accumulated citrate is used to generate NO, ROS and prostaglandins by BMDMs, as well as to produce the antimicrobial metabolite itaconate. (C) Human monocytes shift to a Warburg-like metabolism when stimulated with LPS, but not upon stimulation with the TLR2 ligand P3C. In P3C-stimulated monocytes, increased glycolysis is accompanied by increased OXPHOS. Elevated succinate, itaconate and citrate levels were observed in LPS-stimulated but not in P3C-stimulated monocytes.

Figure 3. Metabolic reprogramming of host cells infected by intracellular bacteria. (A) Salmonella enterica serovar Typhimurium (St) diverts glucose to the bacterial vacuole, while Chlamydia spp. (Chl) induce the Pentose Phosphate Pathway (PPP) to produce nucleotides for bacterial replication. (B) Legionella pneumophila $(L p)$ induces glycolysis, reduces OXPHOS, and might take advantage of this metabolism to acquire amino acids from the host cell. (C) Mycobacterium tuberculosis (Mtb) induces glycolysis, reduces OXPHOS, and increase fatty acids synthesis to use lipid bodies and glycolytic-generated lactate as nutrients for its own growth. 


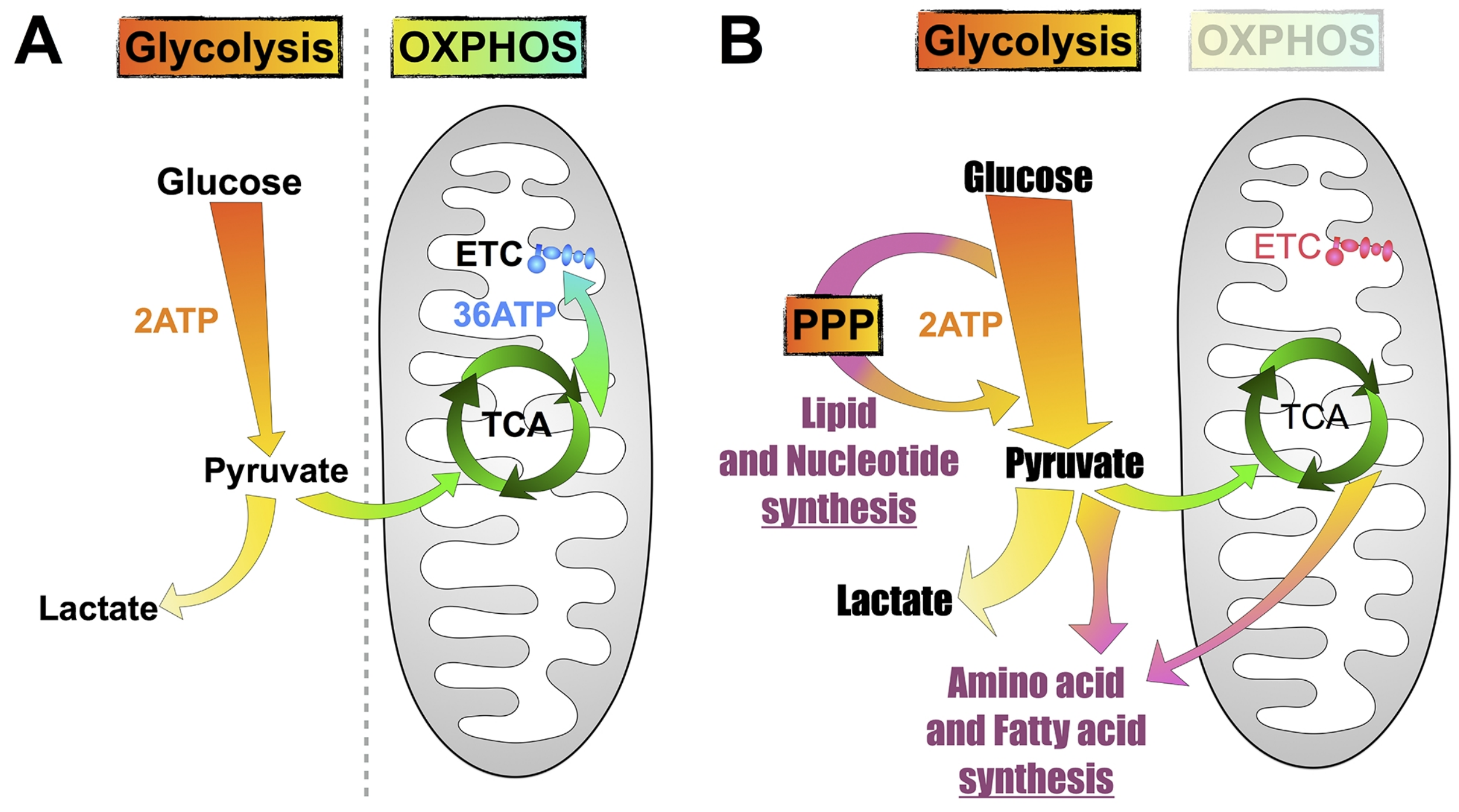



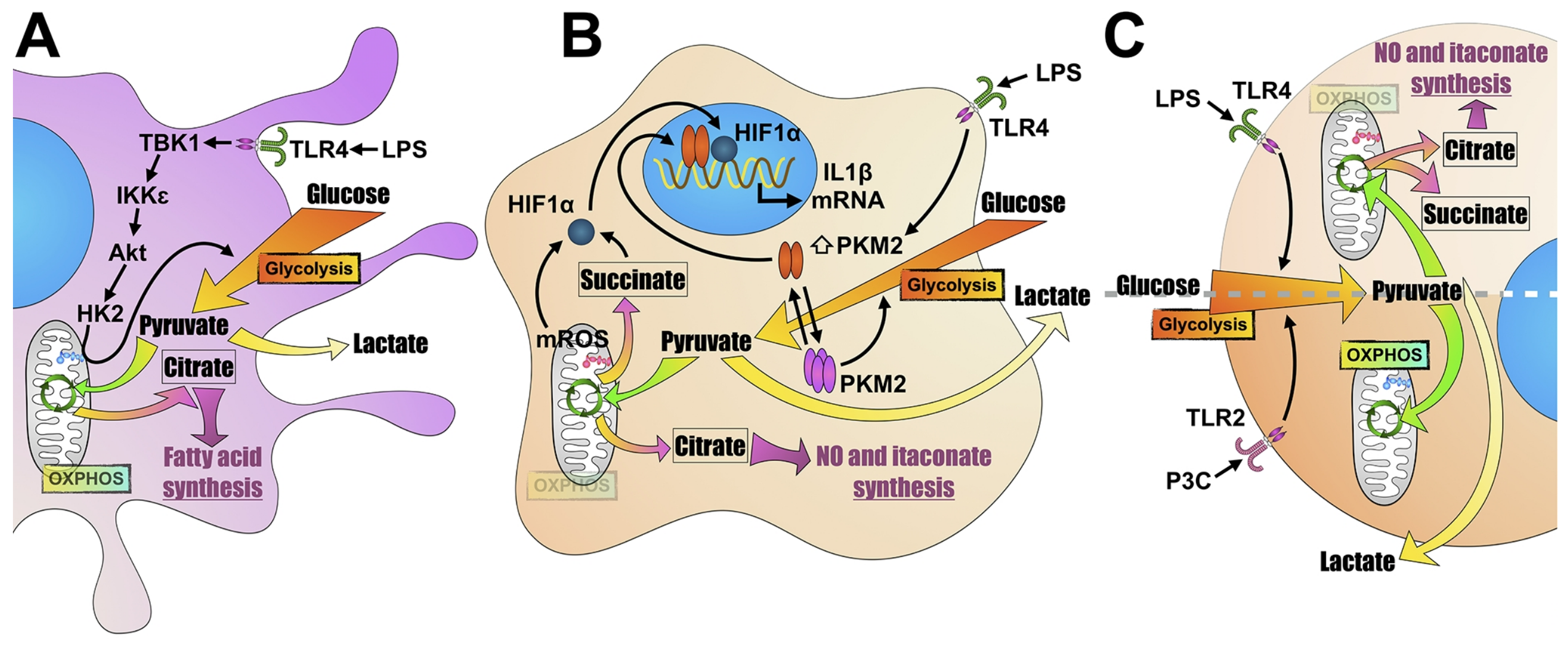
B

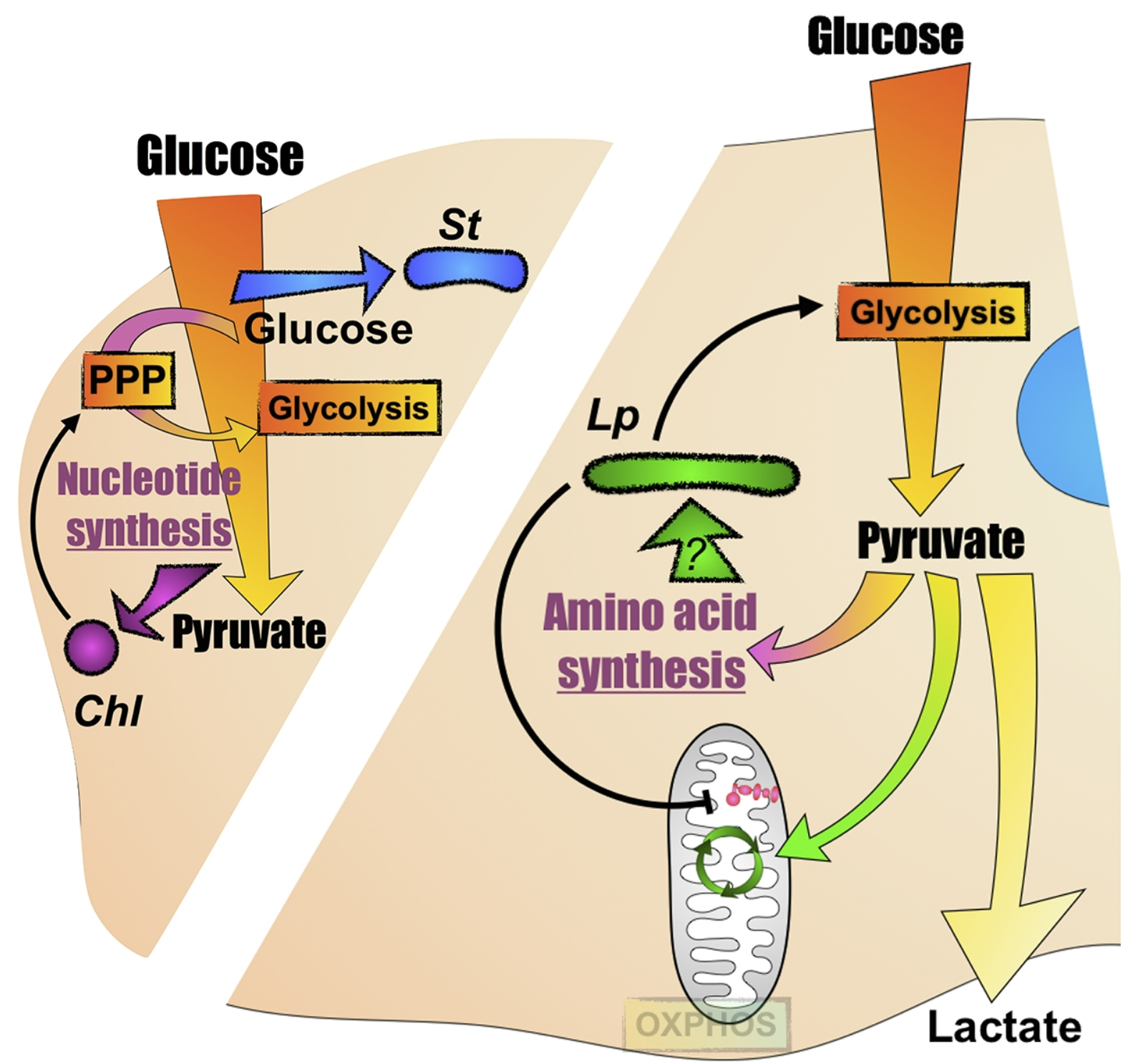

C

\section{Alucose}

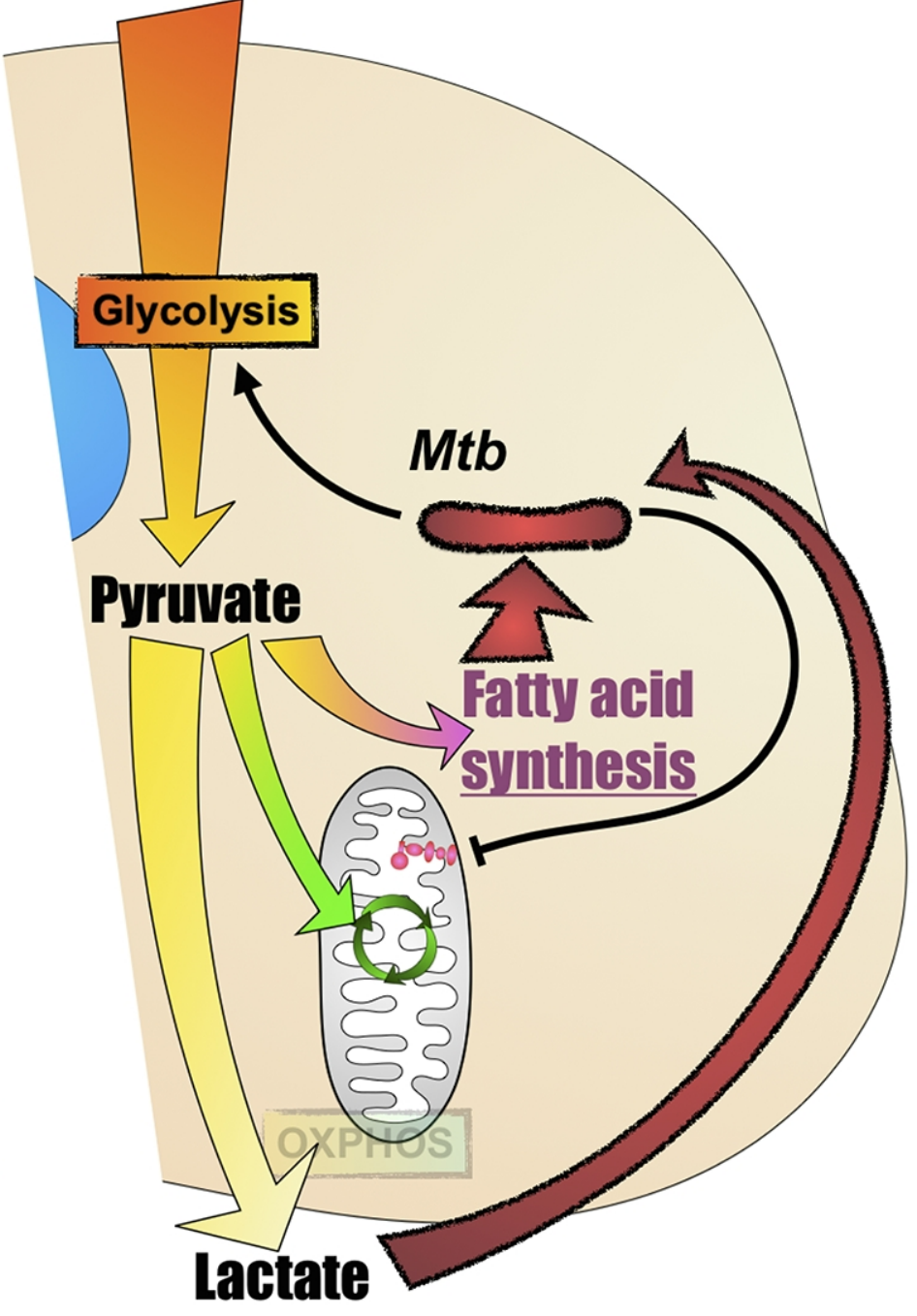

